\title{
CARACTERIZAÇÃO QUÍMICA DO EXTRATO PIROLENHOSO ORIUNDO DA CARBONIZAÇÃO
}

\author{
R. P. VIEIRA ${ }^{1}$, J. B. MOKOCHINSKI ${ }^{2}$, L. C. PALMA ${ }^{3}$, V. G. LIDOINO ${ }^{4}$, M. R. T. \\ HALASZ $^{5}$ \\ ${ }^{1}$ Universidade Estadual de Campinas, Faculdade de Engenharia Química \\ ${ }^{2}$ Universidade Estadual de Campinas, Istituto de Biologia \\ ${ }^{3}$ Universidade Federal do Rio de Janeiro, Programa de Engenharia Química, COPPE \\ ${ }^{4}$ Universidade Federal do Rio de Janeiro, Programa de Engenharia Mecânica, COPPE \\ ${ }^{5}$ Faculdades Integradas de Aracruz, Departamento de Engenharia Química \\ E-mail para contato: rvieira@feq.unicamp.br
}

\begin{abstract}
RESUMO - O extrato pirolenhoso ou extrato ácido é a fração aquosa de compostos orgânicos condensados da fumaça emitida no processo de carbonização. Esta substância apresenta diversas aplicações, como na produção de aromas, aglomerantes, resinas e até a produção de fertilizantes. Cada aplicação depende das características químicas do material. Assim, justifica-se uma prévia caracterização visando identificar os principais componentes do extrato. Devido a isso, este trabalho apresenta a caracterização química de uma amostra de extrato ácido proveniente de uma carbonização de madeira da espécie Eucalyptus Grandis realizada em escala de laboratório em um forno mufla. Dentre as técnicas de caracterização, destaca-se a separação por cromatografia gasosa e a identificação por espectrômetria de massas (CG-EM), em virtude da facilidade de identificação dos componentes por comparação com a biblioteca do equipamento, sendo esta a técnica utilizada neste trabalho. Além disso, também foram determinados o teor de alcatrão, densidade, $\mathrm{pH}$ e acidez. A caracterização mostrou que foi possível obter um extrato dentro dos padrões determinados pela APAN (Associação de Produtores de Agricultura Natural). Os resultados também mostraram que dentre os compostos identificados no CG-EM, destacam-se os fenólicos.
\end{abstract}

\section{INTRODUÇÃO}

Por todo o Brasil, a produção de carvão vegetal ocorre em carvoarias rudimentares e juntas elas atendem uma desmensurada demanda energética. Embora exista toda essa produção ligada ao carvão vegetal, pouco se fala na recuperação dos condensáveis da fumaça e no beneficiamento de seus componentes.

Diversos estudos apontam que apenas uma fração da madeira é convertida em carvão e que grande parte se transforma em gases e vapores (Lu et al., 2012; Liu et al., 2012; Diez et al., 2012; Kijima et al., 2011), tornando interessante estudar a recuperação desse subproduto que, dependendo das características, pode apresentar um alto valor agregado. Este subproduto é popularmente conhecido como bioóleo, que é subdividido em uma parte ácida (extrato ácido 
ou pirolenhoso) e uma parte composta por hidrocarbonetos aromáticos, o chamado alcatrão (Morvoya et al., 2003).

O extrato pirolenhoso vem atraindo a atenção de muitos pesquisadores, haja vista que o seu beneficiamento pode produzir uma gama de produtos (combustíveis, aromas, algomerntes e resinas), tendo destaque a produção de fertilizantes (Garjic e Koch, 2012). Junto a isto, a utilização deste subproduto faz com que haja uma redução significativa nas emissões gasosas.

Tendo em vista a grande empregabilidade dos produtos obtidos neste processo e a importância de um extrato de qualidade, faz-se necessário conhecer as características deste material. Uma forma de caracterizá-lo consiste em obter valores de acidez, densidade, $\mathrm{pH}$ e teor de alcatrão, além da análise química por meio de técnicas de análise instrumental (Spokas et al., 2011; Martins et al., 2007).

O objetivo deste artigo é expor uma maneira simples de caracterização química do extrato pirolenhoso proveniente da carbonização de madeira da espécie Eucalyptus Grandis em um forno mufla. Nesta caracterização serão considerados o teor de alcatrão, a densidade, o $\mathrm{pH}$ e a acidez. Também será realizada uma cromatografia gasosa acoplada à espectrometria de massas (CG-EM).

\section{METODOLOGIA}

\subsection{Obtenção e rendimento}

Em escala de bancada foi utilizado um forno tipo mufla fabricado pela Quimis Aparelhos Científicos (modelo Q318M21) que pode ser visto na Figura 1. Instalou-se um sistema de coleta composto por um condensador de tubo reto e uma proveta para coletar o extrato. $\mathrm{O}$ controle analógico de temperatura do forno foi ajustado para uma temperatura máxima de $400^{\circ} \mathrm{C}$. Após o início da carbonização a temperatura passou a ser registrada a cada 1 minuto, a fim de determinar a taxa de aquecimento do forno. O rendimento do processo foi estimado realizando-se um balanço de massa global.

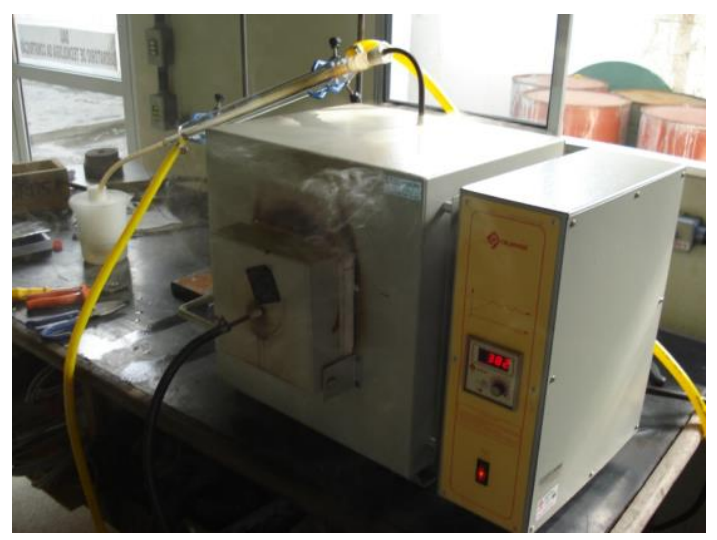

Figura 1 - Carbonização realizada em forno mufla 


\subsection{Caracterização}

$\mathrm{O}$ pH, densidade, teor de alcatrão, acidez total titulável e a identificação dos compostos majoritários foram determinadas usando uma única amostra recolhida durante todo o processo. Previamente, foi realizada uma destilação simples, que além de ter sido usada para determinar o teor de alcatrão, também foi importante para preparar as amostras para a identificação dos compostos em CG-EM. O processo de destilação foi realizado até que todo o líquido presente evaporasse, deixando somente o alcatrão.

O extrato obtido a partir da pirólise do eucalipto foi analisado em cromatógrafo gasoso (Shimadzu QP5050) acoplado a espectrômetro de massas. A separação cromatográfica foi conduzida em coluna analítica capilar DB-5 $(30 \mathrm{~m} \times 0,25 \mathrm{~mm}, 0,25 \mu \mathrm{m})$, de acordo com o seguinte programa de temperatura: 10 minutos sob temperatura constante de $125^{\circ} \mathrm{C}$, seguido de aumento de $8{ }^{\circ} \mathrm{C} / \mathrm{min}$ até 25 minutos, e posterior estabilização em $250^{\circ} \mathrm{C}$ por 15 minutos. O injetor operou no modo split na razão de separação 1:20 (mantendo a temperatura do injetor constante em $250{ }^{\circ} \mathrm{C}$ ). Foi utilizado hélio com gás de arraste, sob fluxo de $1,0 \mathrm{ml} / \mathrm{min}$. A fonte utilizada para ionização da amostra foi de impacto de elétrons (EI) operando em $70 \mathrm{eV}$. A temperatura da interface foi de $280{ }^{\circ} \mathrm{C}$. A aquisição dos espectros foi obtida no intervalo de $m / z 60$ - 500. A identificação dos componentes do extrato foi feita de acordo com comparações entre os espectros das substâncias e a base de dados da biblioteca NIST/2002.

\section{RESULTADOS E DISCUSSÃO}

\subsection{Obtenção e rendimento}

Diversos trabalhos na literatura apontam que entre $290{ }^{\circ} \mathrm{C}$ e $370{ }^{\circ} \mathrm{C}$ ocorre a degradação total da celulose, atingindo a máxima taxa de produção de ácido acético e voláteis em geral a $370{ }^{\circ} \mathrm{C}$ (Roel et al., 2007; Guedes et al., 2010; Cortez et al., 2008). A partir disto, o "set point" do forno em laboratório foi de $400{ }^{\circ} \mathrm{C}$, visando beneficiar esta etapa de carbonização, sendo que a temperatura na qual se encerrou a coleta foi de $382{ }^{\circ} \mathrm{C}$.

A taxa de aquecimento média do sistema foi de $13,95^{\circ} \mathrm{C} / \mathrm{min}$. Este valor enquadra-se como uma pirólise lenta, conforme Cortez et al. (2008), (assim como ocorre em carvoarias convencionais), sendo que esta taxa influencia diretamente no tipo de composto majoritário da carbonização que, neste caso, teoricamente seriam compostos cetônicos, ácidos carboxílicos e compostos fenólicos (Roel et al., 2007).

Com o passar do tempo de carbonização, parte dos voláteis da fumaça condensávamse e eram recolhidos numa proveta, como mostra a Figura 2, visando determinar o rendimento da carbonização neste tipo de forno.

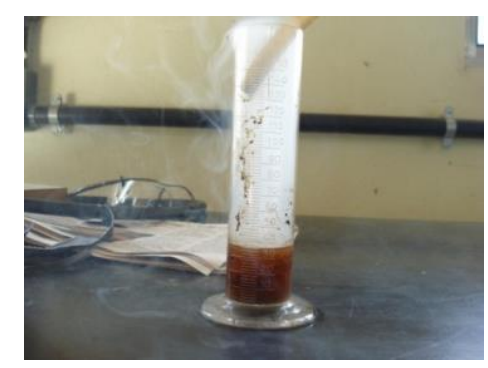

Figura 2 - Extrato pirolenhoso obtido no processo de pirólise lenta em forno mufla. 
Ao fim do processo verificou-se que, das $200 \mathrm{~g}$ de madeira inicialmente adicionadas ao forno, 99,8 $\mathrm{g}$ de carvão foram produzidas, como mostra a Tabela 1.

Tabela 1 - Rendimento da carbonização

\begin{tabular}{|c|c|c|c|}
\hline Madeira & Carvão & Condensado & Gases não condensáveis e perdas \\
\hline $200 \mathrm{~g}$ & $99,8 \mathrm{~g}$ & $34,2 \mathrm{~g}$ & $66 \mathrm{~g}$ \\
\hline $100,00 \%$ & $49,90 \%$ & $17,10 \%$ & $33,00 \%$ \\
\hline
\end{tabular}

Como esperado, atingiu-se um alto rendimento de carvão se comparado aos processos de carbonização realizados nas carvoarias, que é comumente obtido em média $25 \%$. Esse alto rendimento de carvão pode ser explicado pela temperatura do processo que permaneceu em torno de $380{ }^{\circ} \mathrm{C}$. Junto a isto, a atmosfera inerte de argônio faz com que a quantidade de oxigênio no processo fosse bem reduzida, minimizando a combustão de parte da madeira (Antal et al., 2003; Nakai et al., 2007). Além disso, é importante ressaltar que o aquecimento do forno mufla ocorre por uma fonte externa de energia, ao contrário do que ocorre nas carvoarias, ou seja, parte da madeira entra em combustão para fornecer a energia necessária ao processo fazendo com que o rendimento seja menor.

Ainda referindo-se à Tabela 1, percebe-se que o extrato ácido condensado corresponde a $17 \%$ da biomassa utilizada. Esse percentual é baixo, uma vez que o sistema de coleta utilizado não foi o ideal, tendo em vista que diversos trabalhos utilizam outros equipamentos para maximizar a formação de condensados. Por outro lado, o percentual de $33 \%$ de gases não condensáveis está dentro do esperado pela literatura (Cortez et al., 2008).

\subsection{Caracterização}

A caracterização da amostra foi realizada após a destilação, cujo objetivo era eliminar ao máximo o alcatrão solúvel e assim melhorar a qualidade do extrato. A destilação registrou uma temperatura máxima de $137^{\circ} \mathrm{C}$. Essa temperatura refere-se àquela em que somente alcatrão sólido permaneceu no fundo do balão volumétrico. A amostra na sua forma pura e destilada pode ser observada na Figura 3.
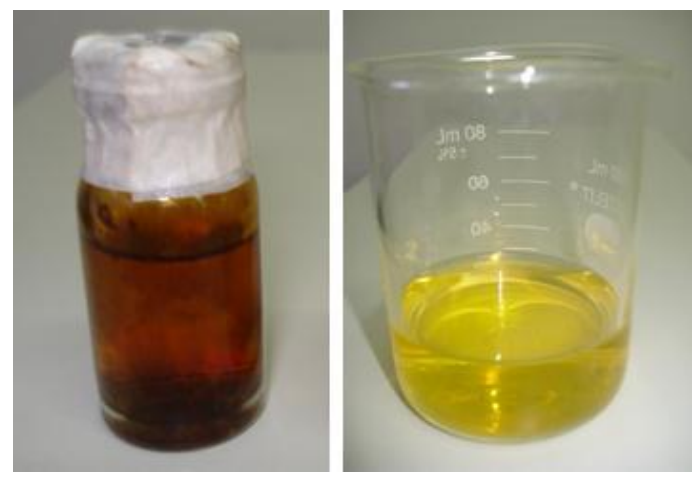

Figura 3 - Amostra do forno mufla pura (à esquerda) e destilada (à direita)

A Tabela 2 contém a massa obtida de extrato isento de alcatrão e o percentual deste na respectiva amostra. 
Tabela 2 - Massa e percentual de alcatrão na amostra

\begin{tabular}{|c|c|c|c|c|c|c|}
\hline \multirow{2}{*}{ Amostra } & \multicolumn{3}{|c|}{ Balão volumétrico(g) } & Amostra & \multicolumn{2}{c|}{ Alcatrão } \\
\cline { 2 - 7 } & Vazio & $\begin{array}{c}\text { Com } \\
\text { amostra }\end{array}$ & $\begin{array}{c}\text { Após a } \\
\text { destilação }\end{array}$ & Massa (g) & Massa (g) & Teor (\%) \\
\hline Mufla & 103,3326 & 109,1776 & 103,3744 & 5,581 & 0,0418 & 0,72 \\
\hline
\end{tabular}

A Tabela 3 fornece a densidade, acidez total e o $\mathrm{pH}$ da amostra de extrato pirolenhoso. Com relação ao $\mathrm{pH}$, a amostra destilada possui $\mathrm{pH}$ igual a 3,2 , considerando um erro de 0,2 no pHmetro.

Tabela 3 - Densidade, acidez total e pH da amostra

\begin{tabular}{|c|c|c|c|c|c|}
\hline \multirow{2}{*}{ Amostra } & \multicolumn{3}{|c|}{ Densidade } & $\begin{array}{c}\text { Acidez total } \\
\text { Titulável (\%) }\end{array}$ & $\begin{array}{c}\mathrm{pH} \\
\text { destilado }\end{array}$ \\
\cline { 2 - 6 } & Massa (g) & Volume (mL) & Valor $(\mathrm{g} / \mathrm{mL})$ & 3,13 & 3,2 \\
\hline Mufla & 34,2 & 33,7 & 1,0148 & 3 & \\
\hline
\end{tabular}

Como pode ser confirmado na Tabela 4, os valores obtidos nesta caracterização estão próximos aos valores normatizados pela APAN (Associação de Produtores de Agricultura Natural). Esta norma aplica-se a extratos pirolenhosos oriundos de fornos de carbonização de madeira ou bambu, feitos de barro ou de tijolos, coletados processados e comercializados sob orientação da APAN, visando melhorar a qualidade em atenção ao mercado consumidor.

Tabela 4 - Caracterização APAN

\begin{tabular}{|c|c|c|}
\hline \multirow{2}{*}{ Características } & \multicolumn{2}{|c|}{ Extrato Pirolenhoso e Pirobambú } \\
\cline { 2 - 3 } & Decantado & Destilado \\
\hline $\mathrm{pH}$ & $3,5 \pm 1,2$ & $3,0 \pm 0,4$ \\
\hline Densidade & $1,007 \sim 1,020$ & $1,002 \sim 1,010$ \\
\hline Acidez $(\%)$ & $2,9 \sim 6,0$ & $1,5 \sim 3,0$ \\
\hline Cor & $\begin{array}{c}\text { Amarelo claro a vermelho } \\
\text { achocolatado }\end{array}$ & Sem cor \\
\hline Transparência & $\begin{array}{c}\text { Transparente, sem material } \\
\text { em suspensão }\end{array}$ & $\begin{array}{c}\text { Transparente, sem material } \\
\text { em suspensão }\end{array}$ \\
\hline $\begin{array}{c}\text { Temperatura de coleta }- \\
\text { fumaça }\left({ }^{\circ} \mathrm{C}\right) \text { (saída chaminé) }\end{array}$ & $80 \sim 120$ & $80 \sim 120$ \\
\hline
\end{tabular}

Fonte: APAN (Associação de Produtores de Agricultura Natural) - 1990

A composição química do extrato pirolenhoso foi determinada por CG-EM. A identidade dos compostos foi assegurada pela correlação dos espectros com a biblioteca, estabelecendo como critério de confirmação valores superiores a $90 \%$. A amostra foi filtrada em membrana $0,22 \mu \mathrm{m}$ para remoção de componentes particulados e posterior derivatização.

Sabe-se que a composição química dos extratos obtidos a partir do processo de pirólise é dependente de características dos materiais de origem bem como das condições de carbonização (Ma et al., 2013). Materiais lenhosos - ricos em lignina e celulose - quando submetidos a reações pirolíticas, são termicamente degradados em cetonas, álcoois, e 
derivados de furano e pireno, podendo também ser convertidos em compostos fenólicos (Wei, Ma e Dong, 2010; Souza et al., 2012). Neste trabalho, o material utilizado como fonte para o preparo do extrato é decorrente do eucalipto, apresentando altas concentrações de ligninas. Ao degradar, esse material forma produtos pirolíticos típicos, tais como: guaiacol, siringol, pirocatecol e outros derivados. A Figura 4 exibe as substâncias mais abundantes identificadas a partir de CG-EM.<smiles>COc1ccccc1O</smiles>

Guaiacol<smiles>Oc1cccc(O)c1O</smiles>

Pirogalol<smiles>Cc1cccc(O)c1</smiles>

Cresol<smiles>COc1ccccc1OC</smiles>

Veratrol<smiles>COc1cccc(OC)c1O</smiles>

Siringol<smiles>COc1cc(C=O)ccc1O</smiles>

Figura 4 - Principais compostos identificados por CG-EM no extrato pirolenhoso.

Vários autores ressaltam o potencial antioxidante do extrato pirolenhoso, principalmente devido à presença de substâncias fenólicas (Wei et al., 2010; Loo et al., 2008; Ma et al., 2011). Os resultados obtidos concordam com os dados reportados pela literatura. A maior parte dos compostos identificados foram fenóis e ácidos orgânicos, confirmando a possibilidade de uso deste extrato como agente antioxidante.

\section{CONCLUSÃO}

A análise por CG-EM permitiu a identificação de ácidos orgânicos e compostos fenólicos característicos do extrato pirolenhoso. Os mais abundantes foram: guaiacol, cresol e siringol. Os resultados indicam que a formação dessas substâncias deve-se a decomposição térmica da lignina em altas temperaturas, ou seja, superiores a $300{ }^{\circ} \mathrm{C}$. Além disso, foi possível realizar uma caracterização relativamente simples e comprovar que a qualidade do extrato produzido em escala laboratorial com controle de temperatura definido, se encontra razoavelmente nas condições básicas determinadas pela APAN.

\section{REFERÊNCIAS}

ANTAL, M. J.; MOCHIDZUKI, K.; PAREDES, L. S. Flash Carbonization of Biomass. Ind. and Eng. Chem. Res., v. 42, p. 3690-3698, 2003.

APAN - Associação de Produtores de Agricultura Natural. Extrato Pirolenhoso Abrangência e objetivos da norma. 
CORTEZ, L. B., LORA, E. S., GÓMEZ, E. O. Biomassa para energia, Campinas, Editora Unicamp, 2008.

DIEZ, M. A.; ALVAREZ, R.; FERNANDEZ, M. Biomass derived products as modifiers of the rheological properties of coking coals. Fuel, v. 96, p. 306-313, 2012.

GAJIC, A.; KOCH, H. J. Sugar beet ( L.) growth reduction caused by hydrochar is related to nitrogen supply. J. of Environm. Qual., v. 41, p. 1067-1075, 2012.

GUEDES, C. L. B; ADÃO, D. C.; QUESSADA, T. P.; BORSATO, D.; GALÃO, O. F.; DI MAURO, E.; PÉREZ, J. M. M; ROCHA, J. D. Avaliação de Biocombustível Derivado do Bio-óleo Obtido por Pirólise Rápida de Biomassa Lignocelulósica como Aditivo para Gasolina. Quim. Nova, v. 33, p. 781-786, 2010.

KIJIMA, M.; HIRUKAWA, T.; HANAWA, F.; HATA, T. Thermal conversion of alkaline lignin and its structured derivatives to porous carbonized materials. Biores. Tech., v. 102, p. 6279-6285, 2011.

LIU, Z. G.; QUEK, A.; HOEKMAN, S. K.; SRINIVASAN, M. P.; BALASUBRAMANIAN, R. Thermogravimetric investigation of hydrochar-lignite co-combustion. Biores. Tech., v. 123, p. 646-652, 2012.

LOO, A.Y.; JAIN, K.; DARAH, I. Antioxidant activity of compounds isolated from the pyroligneous acid, Rhizophora apiculata. Food Chem., v. 107, p. 1151-1160, 2008.

LU, K. M.; LEE, W. J.; CHEN, W. H.; LIU, S. H.; LIN, T. C. Torrefaction and low temperature carbonization of oil palm fiber and eucalyptus in nitrogen and air atmospheres. Biores. Tech., v. 123, p. 98-105, 2012.

MA, C.; SONG, K.; YU, J.; YANG, L.; ZHAO, C.; WANG, W.; ZU, G., ZU, Y. Pyrolysis process and antioxidant activity of piroligneous acid from Rosmarinus officinalis leaves. J. of Anal. and Appl. Pyrolysis, v. 104, p. 38-47, 2013.

MA, X.; WEI, Q.; ZHANG, S.; SHI, L.; ZHAO, Z. Isolation and bioactivities of organic acids and phenols from walnut shell pyroligneous acid. J. of Anal. and Appl. Pyrolysis, v. 91, p. 338-343, 2011.

MARTINS, A. F.; DINIZ, J.; STAHL, J. A. CARDOSO, A. L. Caracterização dos produtos líquidos e do carvão da pirólise de serragem de eucalipto. Quím. Nova, v. 30, p. 873$878,2007$.

MORVOVA, M.; MORVA, I.; JANDA, M.; HANIC, F.; LUKAE, P. Combustion and carbonisation exhaust utilisation in electric discharge and its relation to prebiotic chemistry. Int. J. of Mass Spec., v. 223, p. 613-625, 2003.

NAKAI, T.; KARTAL, S. N.; HATA, T.; IMAMURA, Y. Chemical characterization of pyrolysis liquids of wood-based composites and evaluation of teir bio-efficiency. Build. and Environm., v. 42, p. 1236-1241, 2007. 
ROEL, A. R.; LEONEL, L. A. K.; FAVARO, S. P.; ZATARIM, M.; MOMESSO, C. M. V.; SOARES, M. V. Avaliação de fertilizantes orgânicos na produção de alface em Campo Grande. Sci. Agrar., v. 8, p. 325-329, 2007.

SOUZA, J. B.; RÉ-POPPI, N.; RAPOSO, J. L. Jr. Characterization of Pyroligneous Acid used in Agriculture by Gas Chromatography-Mass Spectrometry. J. Braz. Chem. Soc., v. 23(4), p. 610-617, 2012.

SPOKAS, K. A.; NOVAK, J. M.; STEWART, C. E.; CANTRELL, K. B.; UCHIMIYA, M.; DUSAIRE, M. G.; RO, K. S. Qualitative analysis of volatile organic compounds on biochar. Chemosphere, v. 85, p. 869-682, 2011.

WEI, Q.; MA, X.; DONG, J. Preparation, chemical constituents and antimicrobial activity of pyroligneous acids from walnut tree brances. J. of Anal. and Appl. Pyrolysis, v. 87, p. 24-28, 2010. 